\title{
Design study of compact medical fixed-field alternating-gradient accelerators
}

\author{
T. Misu, Y. Iwata, A. Sugiura, S. Hojo, N. Miyahara, M. Kanazawa, T. Murakami, and S. Yamada \\ National Institute of Radiological Sciences, Anagawa, Inage, Chiba 263-8555, Japan
}

(Received 20 April 2004; published 21 September 2004)

\begin{abstract}
We have studied the various conditions and limitations for achieving compact fixed-field alternatinggradient (FFAG) accelerators to be widely used in heavy-ion cancer therapy. For the case of a normalconducting FFAG accelerator, our linear calculation indicates 12-cell radial sectors with a field index of 10.5 as a suitable configuration. We found that its ring circumference can be as small as $70 \mathrm{~m}$ and that triple-cascade rings are needed to accelerate a carbon beam from $40 \mathrm{keV} / \mathrm{u}$ to $400 \mathrm{MeV} / \mathrm{u}$. In this paper, we report a systematic analysis based on a linear optical model, a comparison of various types of FFAG, and a design example with some technical concerns. An important result is that viable radialsector designs are possible with circumference factor $C$ significantly lower than the value 4.45 previously quoted.
\end{abstract}

DOI: 10.1103/PhysRevSTAB.7.094701

PACS numbers: 29.20.-c, 87.53.-j

\section{INTRODUCTION}

As latest estimates indicate that cancer deaths will increase to $10^{7}$ per year by 2020 , cancer treatment has become one of the top priorities for global health care. For curing localized cancer, the World Health Organization ranks radiation therapy as one of the most important and effective methods. Radiation therapy damages the generic material of malignant cells in tumor tissue and makes it impossible for these cells to reproduce, while the adjacent normal cells are able to repair themselves and function properly.

In recent years, particle-accelerator-based cancer therapy has been actively carried out throughout the world. There exist three facilities around the world carrying out carbon-beam therapy. The Heavy Ion Medical Accelerator in Chiba (HIMAC), as one of the world's state-of-the-art facilities, has shown the therapy's effectiveness after treating over 1600 patients [1]. However, such carbon-beam medical accelerators are rather large in size and expensive, which prohibit the widespread use of carbon-beam radiotherapy. Therefore, there is a need to develop medical carbon-beam accelerators that are compact, low-cost, and simple to operate [2]. We have been studying the possible use of fixed-field alternatinggradient (FFAG) accelerators, which are expected to have potential advantages over conventional synchrotrons. Since the fixed field of FFAG allows a higher magnetic field than synchrotrons, a more compact design may be achieved. The higher repetition rate of FFAG is expected due to its time-independent field structure, while that of a conventional synchrotron is limited by the time required for the pulsed magnetic field cycle. With such a high repetition rate, a highly controlled delivery of the dose can be possible when using the latest irradiation techniques. In the spot-scanning method [3], the spot-size carbon beam needs to be steered quickly in the patient's body by magnetic deflection, while there are no beams being supplied during a transition from one constituent field spot to the next. This type of scanning method can achieve high irradiation accuracy by reducing unwanted dosage and can eliminate the collimator/bolus used in the wobbling method. In the following section of this paper, we discuss the basic parameters of the medical carbonbeam accelerator and conditions for achieving a compact design. Various types of FFAG configuration are analyzed and compared in the framework of linear optics in Sec. III. Section IV explains the accelerator design and its technical concerns.

\section{BASIC PARAMETERS AND STUDY CONDITIONS}

Based on the successful clinical records of HIMAC, we started our design study with the basic beam parameters given in Table I.

Here, the extraction energy was chosen so that the maximum depth of particle penetration in water becomes over $25 \mathrm{~cm}$, sufficient to cover the whole volume of a human body.

With the above parameters, we have further set constraints on design from compactness and manufacturing points of view. As we start the analysis, normal conducting magnets are assumed. Considering the purpose of this project, our design should clearly offer the smallest machine among the existing carbon-beam radiotherapy accelerators. Furthermore, the orbit excursion in the FFAG should be kept as small as possible, mainly for manufacturing reasons. The shunt impedance of the untuned

TABLE I. FFAG basic parameters.

\begin{tabular}{lc}
\hline \hline Particle at injection/extraction & $\mathrm{C}^{4+} / \mathrm{C}^{6+}$ \\
Energy $(\mathrm{MeV} / \mathrm{u})$ & $0.04-400$ \\
Number of particles $(\mathrm{pps})$ & $2 \times 10^{9}$ \\
Repetition rate $(\mathrm{Hz})$ & 200 \\
\hline \hline
\end{tabular}


broadband cavity decreases with the orbit excursion when loading high-permeability metallic alloy (MA) cores inside the cavity. No MA cores, which can cover an orbit excursion larger than $1 \mathrm{~m}$, have been manufactured so far. The ceramic spacer for the accelerating gap of the $\mathrm{rf}$ cavity cannot be manufactured any larger than $1 \mathrm{~m}$ in diameter at present.

We have therefore assigned the following additional constraints: (i) a maximum magnetic field of $1.9 \mathrm{~T}$, (ii) a ring circumference of $70 \mathrm{~m}$ or less (ring radius of $11 \mathrm{~m}$ or less), (iii) an orbit excursion of $1 \mathrm{~m}$ or less, and (iv) a length of straight section per cell of $1 \mathrm{~m}$ or more.

\section{ANALYSIS ON VARIOUS TYPES OF FFAG CONFIGURATION}

\section{A. General description}

Studies and developments of fixed-field alternatinggradient focusing machines have been rare since the group of MURA Universities constructed the first FFAG model in late 1950s [4]. In this section, we briefly review the general characteristics of the FFAG principle.

FFAG accelerators are known to possess prominent nonlinear focusing forces. They are generally classified into the following two types: radial sector and spiral sector. In both types, the average field $\langle B\rangle$ increases with radius $R$ as expressed by

$$
\langle B\rangle=\left\langle B_{0}\right\rangle\left(R / R_{0}\right)^{k} .
$$

Here $\left\langle B_{0}\right\rangle$ is the average field at some reference radius $R_{0}$ and the field index $k$ is a constant.

For the radial-sector type, the magnetic fields have opposite directions in alternating focusing and defocusing magnets. Strong radial focusing and axial defocusing forces are present in the focusing $(F)$ magnets, while the forces act opposite in the defocusing $(D)$ magnets. The proper combination of $F$ and $D$ magnets guarantees orbit stability, even though the presence of reverse fields consequently results in large circumference.

Unlike the radial-sector type, the spiral sector can have a smaller circumference because its magnetic field is unidirectional. Since the particles pass through the magnet edges at an angle other than $90^{\circ}$, strong quadratic forces are produced. By keeping a constant magnetic flutter and a constant spiral angle for all momenta, the focusing actions become independent of radius.

These types of accelerators are often called scaling FFAGs due to the constant shape of the equilibrium orbits and tunes. Such a scaling property is advantageous to avoid resonance buildup of betatron oscillations and widen momentum range of the machine.

Since the first proton FFAG accelerator [5] was constructed by the KEK group a few years ago, there has been growing interest in FFAG accelerators for several different applications, such as high-intensity proton sources, muon acceleration/cooling, and cancer therapy.
For instance, a $150 \mathrm{MeV}$ proton FFAG accelerator [6] has recently been constructed at KEK as a prototype for proton beam therapy and other various applications. The construction of three-stage FFAG accelerators is underway at Kyoto University Research Reactor Institute to carry out the basic studies on accelerator-driven subcritical reactor [7]. Both scaling FFAGs and nonscaling FFAGs with rapid acceleration have been also proposed as intense muon sources [8,9]. This paper discusses the possible medical use (carbon-beam radiotherapy) of FFAG accelerators by systematically exploring the optical parameters to achieve a compact design.

\section{B. Linear optical model}

We developed a linear optical calculation code in order to carry out systematic analysis on both radial- and spiral-sector FFAGs for a compact design. Lattice structure can be generalized in terms of three basic elements as in Fig. 1. Here, $\Theta_{L}, \Theta_{F}$, and $\Theta_{D}$ are the open angles of the drift space, and the focusing and defocusing magnets, respectively. $\theta_{F}$ and $\theta_{D}$ are the bending angles of $F$ and $D$ magnets. The radii $r_{1}$ and $r_{2}$ are the distances from the center of the machine to the beam orbit at each boundary. $\phi_{1}$ and $\phi_{2}$ are the angles between the beam orbit and a normal vector at each boundary.

Since the size of a circular accelerator depends on its maximum magnetic field strength, one can define reduced parameters with respect to the radius of curvature at maximum momentum, denoted by $\rho_{0}$. For each optical element in the radial-sector type, these reduced parameters can be defined in the following relations.

\section{Drift space}

The relation among geometrical angles is given by $\phi_{2}=\Theta_{L}+\phi_{1}$.

One can derive the reduced parameters of both $r_{1}$ and $r_{2}$ with respect to the reference radius of curvature $\rho_{0}$ in terms of geometrical angles

$$
\frac{r_{1}}{\rho_{0}}=\frac{\cos \left(\Theta_{L}+\phi_{1}\right)}{\sin \Theta_{L}}\left(\frac{\lambda}{\rho_{0}}\right)
$$

and

$$
\frac{r_{2}}{\rho_{0}}=\frac{1}{\cos \Theta_{L}-\sin \Theta_{L} \tan \phi_{1}}\left(\frac{r_{1}}{\rho_{0}}\right) .
$$

\section{Focusing ( F) magnet}

Similar relations also hold for the focusing magnet: $\varepsilon_{1}=\phi_{1} ; \varepsilon_{2}=\theta_{F}-\phi_{1}-\Theta_{F} ; \phi_{2}=-\varepsilon_{2}$. Here, $\varepsilon_{1}$ and $\varepsilon_{2}$ are the edge angles at the element boundary.

With respect to the radius of curvature $\rho_{F}$, the reduced parameters in the focusing magnet section are found as 


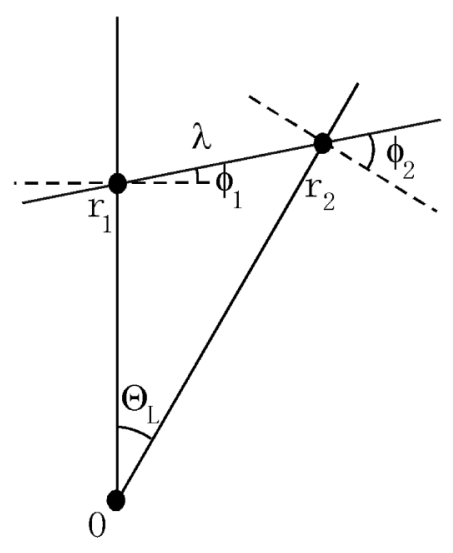

Drift Space

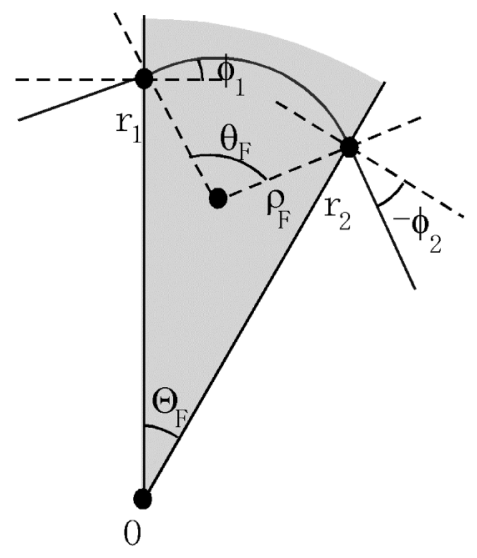

Focusing Magnet

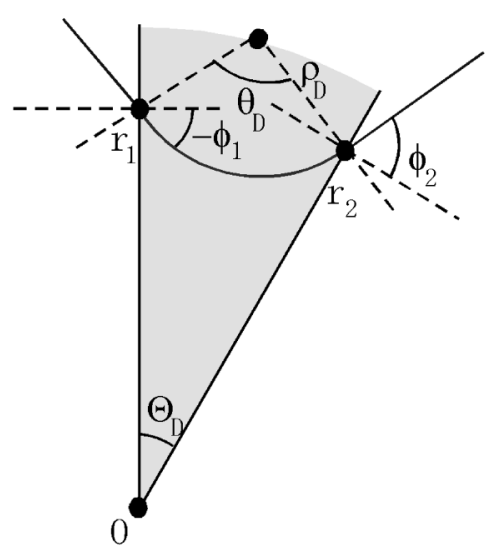

Defocusing Magnet

(a)

(b)

(c)

FIG. 1. Basic elements of a radial-sector FFAG.

$$
\begin{aligned}
\frac{r_{1}}{\rho_{F}}= & \frac{1}{\tan \Theta_{F}}\left[\sin \phi_{1}+\sin \left(\theta_{F}-\phi_{1}\right)\right] \\
& +\left[\cos \phi_{1}-\cos \left(\theta_{F}-\phi_{1}\right)\right]
\end{aligned}
$$

and

$$
\frac{r_{2}}{\rho_{F}}=\frac{1}{\sin \Theta_{F}}\left[\sin \phi_{1}+\sin \left(\theta_{F}-\phi_{1}\right)\right] .
$$

By rescaling the above parameters in terms of the reference radius of curvature $\rho_{0}$ instead, we get

$$
\begin{aligned}
\frac{r_{1}}{\rho_{0}}= & \left\{\frac{1}{\tan \Theta_{F}}\left[\sin \phi_{1}+\sin \left(\theta_{F}-\phi_{1}\right)\right]+\left[\cos \phi_{1}\right.\right. \\
& \left.\left.-\cos \left(\theta_{F}-\phi_{1}\right)\right]\right\}\left(\frac{\rho_{F}}{\rho_{0}}\right)
\end{aligned}
$$

and

$$
\frac{r_{2}}{\rho_{0}}=\frac{1}{\sin \Theta_{F}}\left(\frac{\rho_{F}}{\rho_{0}}\right)\left[\sin \phi_{1}+\sin \left(\theta_{F}-\phi_{1}\right)\right]
$$

\section{Defocusing (D) magnet}

The relations among geometrical angles are given by $\varepsilon_{1}=-\phi, \varepsilon_{2}=\theta_{D}+\phi_{1}+\Theta_{D}$, and $\phi_{2}=\varepsilon_{2}$. The reduced parameters with respect to the radius of curvature $\rho_{D}$ are derived as

$$
\begin{aligned}
\frac{r_{1}}{\rho_{D}}= & \frac{1}{\tan \Theta_{D}}\left[\sin \left(\theta_{D}+\phi_{1}\right)-\sin \phi_{1}\right] \\
& +\left[\cos \left(\theta_{D}+\phi_{1}\right)-\cos \phi_{1}\right]
\end{aligned}
$$

and

$$
\frac{r_{2}}{\rho_{D}}=\frac{1}{\sin \Theta_{D}}\left[\sin \left(\theta_{D}+\phi_{1}\right)-\sin \phi_{1}\right]
$$

Thus,

$$
\begin{aligned}
\frac{r_{1}}{\rho_{0}}= & \left\{\frac{1}{\tan \Theta_{D}}\left[\sin \left(\theta_{D}+\phi_{1}\right)-\sin \phi_{1}\right]\right. \\
& \left.+\left[\cos \left(\theta_{D}+\phi_{1}\right)-\cos \phi_{1}\right]\right\}\left(\frac{\rho_{D}}{\rho_{0}}\right)
\end{aligned}
$$

and

$$
\frac{r_{2}}{\rho_{0}}=\frac{1}{\sin \Theta_{D}}\left[\sin \left(\theta_{D}+\phi_{1}\right)-\sin \phi_{1}\right]\left(\frac{\rho_{D}}{\rho_{0}}\right) .
$$

Therefore, any arbitrary arrangement of these elements can be expressed in terms of the above parameters. If we label all the elements in one cell from one to $n$, its closed orbit can be found by imposing $r_{2} /\left.\rho_{0}\right|_{i}=r_{1} /\left.\rho_{0}\right|_{i+1}(i$ corresponds to the $i$ th element), $r_{2} /\left.\rho_{0}\right|_{n}=r_{1} /\left.\rho_{0}\right|_{1}$, and $\left.\phi_{2}\right|_{i}=\left.\phi_{1}\right|_{i+1}$.

For the spiral-sector type with spiral angle $\xi$, its closed orbit can be obtained by simply replacing the above edge angles by

$$
\varepsilon_{1} \rightarrow \varepsilon_{1}+\sigma_{M} \cdot \xi \quad \text { and } \quad \varepsilon_{2} \rightarrow \varepsilon_{2}-\sigma_{M} \cdot \xi,
$$

where $M$ denotes $F$ or $D$ magnet; $\sigma_{F}=1$ and $\sigma_{D}=-1$.

In terms of the above generalized expressions for both radial- and spiral-sector FFAG accelerators, we can design any lattice configurations in principle. Once the closed orbit of an arbitrary configuration is determined, its accelerator size can be measured by the circumference factor $C$ as

$$
C=\frac{\langle r\rangle}{\rho_{0}}=\left.\sum_{i}^{N_{F}}\left(\frac{\rho_{F}}{\rho_{0}}\right)_{i} \theta_{F}\right|_{i}+\left.\sum_{i}^{N_{D}}\left(\frac{\rho_{D}}{\rho_{0}}\right)_{i} \theta_{D}\right|_{i}+\sum_{i}^{N_{\lambda}}\left(\frac{\lambda}{\rho_{0}}\right)_{i}
$$


(a) Singlet

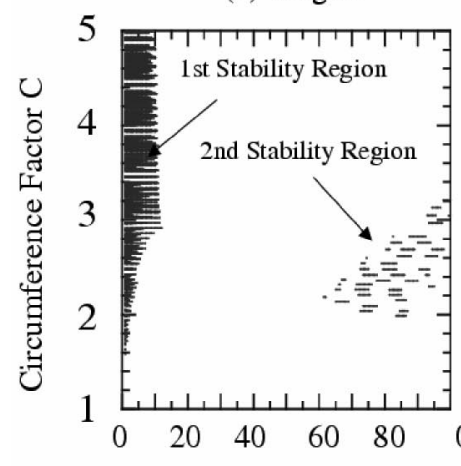

(b) Doublet

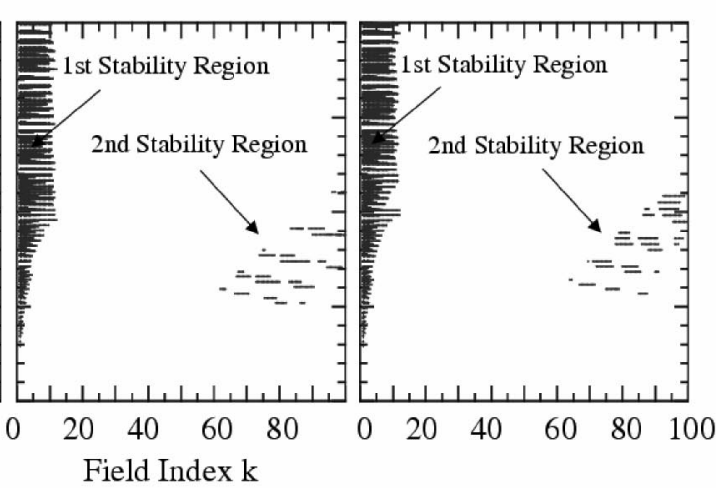

FIG. 2. Stability islands of the 12 cell radial-sector type are compared in the $k$ - $C$ plane for three different configurations: (a) singlet, (b) doublet, and (c) $D-F-D$ triplet. Similar stability regions are observed for all three configurations.

In this approach, we assumed a constant radius of curvature along an equilibrium orbit for each magnet. Once the cell number, the lattice arrangement, and the field index are specified, all the necessary lattice parameters are determined in parameter space, e.g., in terms of $\theta_{F}, \Theta_{F}$, and $\Theta_{L}$, by multiplying the appropriate linear matrices for drift space, gradient sector magnets, and edge focusing.

\section{Radial-sector type}

Using the above optical model, we searched the parameter space by varying the values of $\theta_{F}, \Theta_{F}$, and $\Theta_{L}$ in order to look for the stability regions of three different types of radial-sector configuration for a comparison: singlet configuration [10] in which $F$ and $D$ magnets are placed separately, $F-D$ doublet configuration in which $F$ and $D$ magnets are combined together to create a usable long straight section for placing components such as rf accelerating cavities, and $D-F-D$ triplet configuration [5] in which $F$ and $D$ magnets are combined in one to create a long straight section while reducing the meandering of the orbital path compared to that of the $F-D$ doublet configuration. In Fig. 2, the stability regions for the case of 12 cells are presented in the $k-C$ plane. Here, all the geometrical angles and the field index $k$ have been varied in steps of $1^{\circ}$ and 0.5 , respectively. More than one stability region is observed in the figure, as indicated analytically by the Hill equations obtained at the limit of small-amplitude oscillations. The stability "islands" are well separated and have a similar appearance for all three configurations. However, the higher order stability islands seen at large field indices are associated with large phase advances and betatron oscillations (see Fig. 3), implying that these regions are very sensitive to imperfections and such high field-gradient magnets may not be realized in practice. Thus, we carried out the study for the first stability region.

The theoretical minimum value of $C$ for a large number of radial sectors with no space between them is expected to be 4.45 as derived in Ref. [10], implying the minimum ring radius of about $15 \mathrm{~m}$ for the case of our carbon-beam radiotherapy machine and never satisfying the constraint of ring radius $\leq 11 \mathrm{~m}$ ( or $C \leq 3.3$ ). In our calculation, however, which includes finite drift spaces, a circumference factor of less than 3.0 can be achieved for $k \leq 12$. The radial-sector type, which has not yet been realized for $C$ values in this range, is now seen to offer this capability.

Figure 4 depicts the ring radii with a maximum magnetic field of $1.9 \mathrm{~T}$ calculated for the first stability regions of 8,12 , and 16 cells. Here, we have selected the doublet type so that larger drift spaces can be secured for installation of equipment, since the optical properties are found to be similar for all three configurations. The maximum

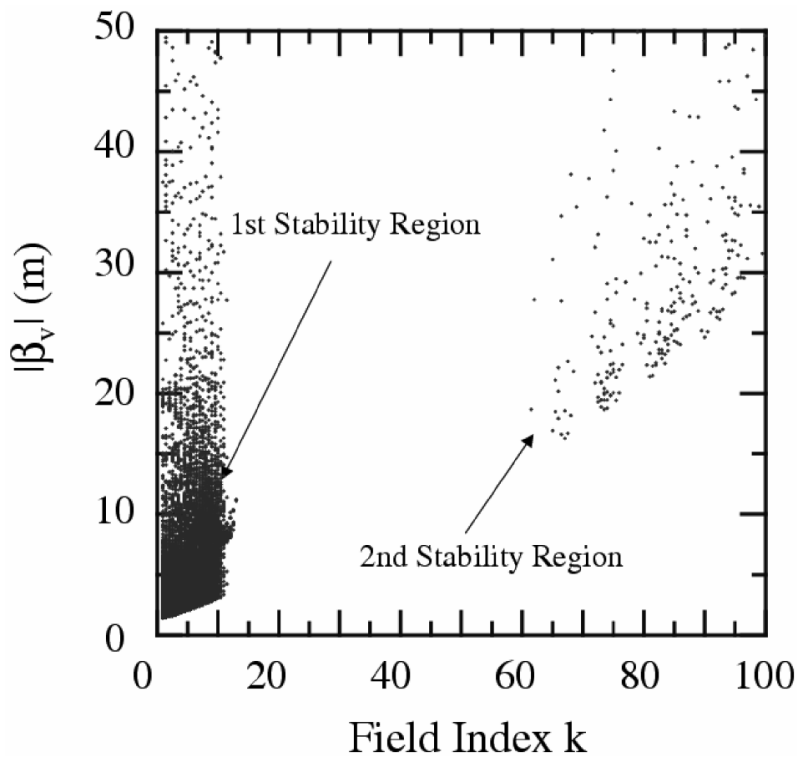

FIG. 3. Magnitudes of vertical $\beta$ function for the case of the 12-cell singlet configuration. The higher order stability islands are associated with large phase advances and betatron oscillations. 


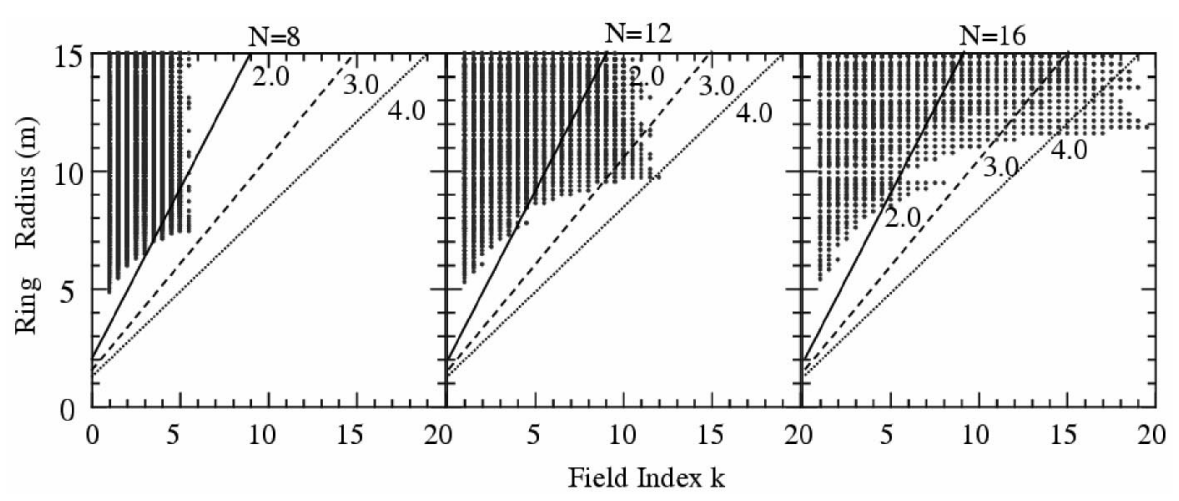

FIG. 4. Ring radii calculated for the first stability region of 8, 12, and 16 cells. Doublet radial-sector configurations are assumed. Straight lines correspond to momentum ratios of 2.0,3.0, and 4.0, respectively, where orbit excursions are less than $1 \mathrm{~m}$.

field indices satisfying the stability conditions for 8,12 , and 16 cells increase with $k$ and reach their maximum values around 6.0, 12.0, and 20.0, respectively. These are consistent with the "necktie" diagram [11] in which the stability regions are plotted in terms of scaled variables of $k / N^{2}$ for given values of the field index $k$ and cell number $N$. In this figure, we also note that the minimum ring radii increase with $k$. Although it is desirable to achieve smaller values of $C$ by lowering the $k$ values, lowering $k$ consequently leads to larger orbit excursion as analytically expressed by

$$
\left(\left\langle r_{\text {ext }}\right\rangle-\left\langle r_{\text {inj }}\right\rangle\right) /\left\langle r_{\text {ext }}\right\rangle=1-\left(p_{\text {inj }} c / p_{\text {ext }} c\right)^{1 /(k+1)} .
$$

The lattice structures of 12 cells show a wider stability region with higher values of the field index than those of eight cells, while retaining the ring circumference below $70 \mathrm{~m}$. Since the momentum excursion of the machine is large, we expect a multiple cascade configuration and Fig. 4 shows several injection momenta which fulfill the orbit excursion of $1 \mathrm{~m}$ or less for a $400 \mathrm{MeV} / \mathrm{u}$ ring. For each injection momentum with ring radius of $11 \mathrm{~m}$ or less, the lengths of drift space per cell are plotted as a function of orbit excursion in Fig. 5. In order to satisfy both orbit excursion and drift space constraints, a momentum ratio of less than 3.0 is clearly preferred and we chose the injection energy of the final cascade ring as $100 \mathrm{MeV} / \mathrm{u}$ (corresponding to a momentum ratio of 2.15) with 12 cells. By imposing the extraction energy of the injector ring prior to the final one to be $100 \mathrm{MeV} / \mathrm{u}$, its injection energy was determined as $6 \mathrm{MeV} / \mathrm{u}$ from a similar argument. Here, the energy of $6 \mathrm{MeV} / \mathrm{u}$ corresponds to the charge-exchange threshold from $\mathrm{C}^{4+}$ to $\mathrm{C}^{6+}$. The same argument was followed to finally construct the triple-cascade design as discussed in the following section. For the low-energy (LE) ring, the number of cells was reduced to ten in order to secure a space for components such as the rf cavities.

\section{Spiral-sector type}

Unlike radial sectors, minimizing $C$ is no longer a major consideration for the spiral-sector type. Multiple stability regions are also observed for the spiral-sector configuration. For the case of a normal-conducting radial-sector type, triple-cascade design was necessary. Here, we search for a double-cascade scheme, i.e., impos-

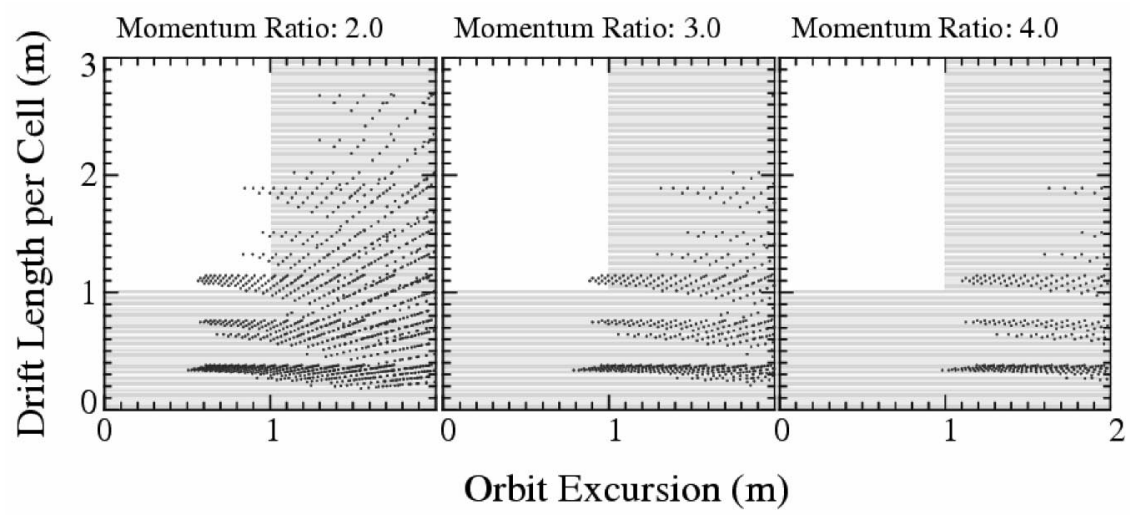

FIG. 5. Lengths of drift space per cell as a function of orbit excursion. Here, all the solutions satisfying a ring radius of less than $11 \mathrm{~m}$ are plotted. For momentum ratios above 3.0, fulfilling the constraints of both orbit excursion and drift length may not be possible. 


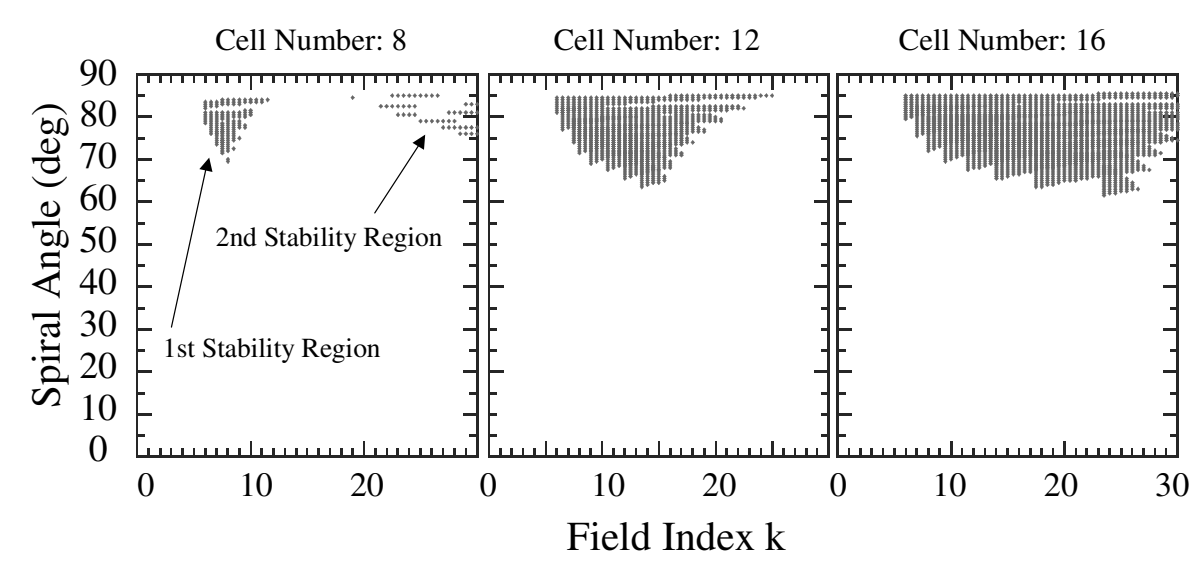

FIG. 6. Required spiral angles for $8,12,16$ cells to fulfill the condition for orbit excursion of $1 \mathrm{~m}$ or less.

ing an injection energy of $6 \mathrm{MeV} / \mathrm{u}$ and an extraction energy of $400 \mathrm{MeV} / \mathrm{u}$ for the main ring, since more compact ring design is expected for spiral-sector FFAG. In Fig. 6, the necessary spiral angles for 8, 12, 16 cells to fulfill the condition for orbit excursion of $1 \mathrm{~m}$ or less are plotted as a function of the field index. In order to design a double-cascade spiral-sector FFAG, the figure indicates that a spiral angle $\xi$ of at least $65^{\circ}$ is required.

As demonstrated in Ref. [2], the size of the stability region for spiral-sector FFAGs depends on a quantity proportional to $\tan \xi$, implying that the stability at higher values of the spiral angle is sensitive to field errors. In such cases, the production of field gradient by adjusting the shape of the magnet pole cannot be realized in practice due to the large fringing field. Together with the fact that the magnet complexity increases considerably and securing the space for the rf cavities becomes a difficult task for highly spiraled FFAG, we therefore omit further discussion of spiral-sector configuration in this report.

\section{DESIGN OF TRIPLE-CASCADE RADIAL- SECTOR FFAG}

Design of a compact ring and small-size components is essential to reduce the manufacturing complexity and cost. The following design is intended to illustrate the features and limitations of a compact radial-sector FFAG for the use of cancer therapy.

\section{A. Lattice design}

Based on the above analysis, we chose a triple-cascade scenario for the radial-sector FFAG, in which each ring has an orbit excursion of less than $80 \mathrm{~cm} . \mathrm{C}^{4+}$ of $40 \mathrm{keV} / \mathrm{u}$ is produced by ECR ion source specified in Table II and directly injected into the low-energy FFAG ring. The LE-FFAG accelerates $\mathrm{C}^{4+}$ up to $6 \mathrm{MeV} / \mathrm{u}$ and then increases the charge-to-mass ratio by a stripping foil placed along the extraction line. $\mathrm{C}^{6+}$ beam is delivered to the medium-energy (ME) FFAG ring and accelerated up to $100 \mathrm{MeV} / \mathrm{u}$. Finally, the high-energy (HE) FFAG ring accelerates $\mathrm{C}^{6+}$ ions up to an energy of $400 \mathrm{MeV} / \mathrm{u}$ and delivers them to clinical treatment rooms for radiotherapy. Overall specification of the system is given in Table III. To reduce the area occupied by the machine as much as possible, we place both LE and ME rings inside the HE ring. In Table IV we roughly estimated the beam transmission rate at each process. Here, the transmission rate during the LE-FFAG acceleration is determined as low as $20 \%$ due to the dynamic aperture of the LE-FFAG, as explained later.

Since the LE ring is rather compact, securing a large enough drift space for the rf cavities is almost impossible and we therefore reduced the lattice periodicity to 5 instead of 10, as shown in Table V. An injection beam pulse width of $1.4 \mu$ s and one-turn injection scheme are enough to achieve the desired beam intensity for the LE ring. The charge stripping foil is placed right before the extraction septum magnet, in order to reduce its magnetic rigidity.

Design of the ME ring is limited by the physical constraint that the inner radius of the ring must be larger than the outer radius of the LE ring while its outer radius is less than the inner radius of the HE ring. For this reason, we chose $k=10.5$ with the maximum magnetic field of $1.45 \mathrm{~T}$ as listed in Table VI.

As explained earlier, the size of the HE ring is solely determined by the maximum magnetic field strength and circumference factor. While keeping the circumference of the ring below $70 \mathrm{~m}$, we also intended to shrink the orbit

TABLE II. ECR ion source specification.

\begin{tabular}{ll}
\hline \hline Particle & $\mathrm{C}^{4+}$ \\
Energy $(\mathrm{keV} / \mathrm{u})$ & 40 \\
Pulse width $(\mathrm{ns})$ & 250 \\
Repetition rate $(\mathrm{Hz})$ & 200 \\
Beam current $(\mu \mathrm{m})$ & 200 \\
Normalized emittance $(\pi \mathrm{mm}$ mrad$)$ & 1 \\
Extraction voltage $(\mathrm{kV})$ & 25 \\
Accelerating gap voltage $(\mathrm{kV})$ & 95 \\
\hline \hline
\end{tabular}




\begin{tabular}{|c|c|c|c|c|c|c|}
\hline \multirow[b]{3}{*}{ Parameter } & \multirow{2}{*}{\multicolumn{2}{|c|}{ Low-energy FFAG }} & \multirow{2}{*}{\multicolumn{2}{|c|}{ Medium-energy FFAG }} & \multirow{2}{*}{\multicolumn{2}{|c|}{ High-energy FFAG }} \\
\hline & & & & & & \\
\hline & Injection & Extraction & Injection & Extraction & Injection & Extraction \\
\hline$T(\mathrm{MeV} / \mathrm{u})$ & 0.04 & 6 & 6 & 100 & 200 & 400 \\
\hline$e / m$ & \multicolumn{2}{|c|}{$1 / 3$} & \multicolumn{2}{|c|}{$1 / 2$} & \multicolumn{2}{|c|}{$1 / 2$} \\
\hline$B \rho(\mathrm{Tm})$ & 0.08634 & 1.0595 & 0.7064 & 2.9554 & 2.9554 & 6.3467 \\
\hline$p / p_{0}$ & 1 & 12.27 & 1 & 4.18 & 1 & 2.15 \\
\hline$k$ & \multicolumn{2}{|c|}{6.5} & \multicolumn{2}{|c|}{10.5} & \multicolumn{2}{|c|}{10.5} \\
\hline$R / R_{0}$ & 1 & 1.43 & 1 & 1.16 & 1 & 1.07 \\
\hline$\varepsilon_{x, y}(\pi \mathrm{mm} \mathrm{mrad})$ & 108 & 8.8 & 8.8 & 2.1 & 2.1 & 0.98 \\
\hline$f_{\text {rev }}(\mathrm{MHz})$ & 0.215 & 1.878 & 0.909 & 3.050 & 2.025 & 3.151 \\
\hline$\langle R\rangle(\mathrm{m})$ & 2.06 & 2.87 & 5.93 & 6.72 & 10.12 & 10.82 \\
\hline
\end{tabular}

TABLE IV. Estimated beam transmission rate.

\begin{tabular}{lccc}
\hline \hline \multicolumn{1}{c}{ Component } & Process & Efficiency $(\%)$ & Required particle number \\
\hline ECR ion source & & & $8.6 \times 10^{10}$ \\
Beam transport $(40 \mathrm{keV} / \mathrm{u})$ & Transmission & 90 & $7.8 \times 10^{10}$ \\
Low-energy FFAG & Injection & 40 & $3.1 \times 10^{10}$ \\
& Acceleration & 20 & $6.2 \times 10^{9}$ \\
Charge stripping foil & Stripping & 95 & $5.9 \times 10^{9}$ \\
& Extraction & 90 & $5.3 \times 10^{9}$ \\
Beam transport $(6 \mathrm{MeV} / \mathrm{u})$ & Transmission & 95 & $5.0 \times 10^{9}$ \\
Medium-energy FFAG & Injection & 90 & $4.5 \times 10^{9}$ \\
& Acceleration & 80 & $4.1 \times 10^{9}$ \\
Beam transport $(100 \mathrm{MeV} / \mathrm{u})$ & Extraction & 90 & $3.4 \times 10^{9}$ \\
High-energy FFAG & Transmission & 95 & $3.2 \times 10^{9}$ \\
& Injection & 90 & $2.9 \times 10^{9}$ \\
Beam transport $(400 \mathrm{MeV} / \mathrm{u})$ & Extraction & 80 & $2.3 \times 10^{9}$ \\
\hline \hline
\end{tabular}

TABLE V. Basic lattice parameters of the LE-FFAG ring.

\begin{tabular}{|c|c|c|}
\hline & Injection & Extraction \\
\hline Energy $T(\mathrm{MeV} / \mathrm{u})$ & 0.04 & 6 \\
\hline Number of cells $N$ & \multicolumn{2}{|c|}{10} \\
\hline Periodicity & \multicolumn{2}{|c|}{5} \\
\hline Field index $k$ & \multirow{2}{*}{\multicolumn{2}{|c|}{$\begin{array}{c}6.5 \\
(3.16,0.90)\end{array}$}} \\
\hline Tune (horizontal, vertical) & & \\
\hline Ring circumference $(\mathrm{m})$ & 13.167 & 18.404 \\
\hline Frequency (MHz) & 0.215 & 1.878 \\
\hline Acceleration voltage $(\mathrm{kV})$ & \multicolumn{2}{|c|}{3.3} \\
\hline Minimum radius (m) & 1.956 & 2.720 \\
\hline Maximum radius (m) & 2.160 & 3.019 \\
\hline Length of long straight (m) & 0.718 & 1.003 \\
\hline Length of short straight (m) & 0.153 & 0.214 \\
\hline Field strength $(F)(\mathrm{T})$ & 0.153 & 1.347 \\
\hline Field strength $(D)(\mathrm{T})$ & 0.182 & 1.608 \\
\hline Sector angle $\Theta_{F}(F)(\operatorname{deg})$ & \multicolumn{2}{|c|}{16} \\
\hline Bending angle $\theta_{F}(F)(\mathrm{deg})$ & \multicolumn{2}{|c|}{$58.8,42.1$} \\
\hline Sector angle $\Theta_{D}(\mathrm{D})(\mathrm{deg})$ & \multicolumn{2}{|c|}{1} \\
\hline Bending angle $\theta_{D}(\mathrm{D})$ (deg) & \multicolumn{2}{|c|}{$17.0,11.9$} \\
\hline
\end{tabular}

TABLE VI. Basic lattice parameters of the ME-FFAG ring.

\begin{tabular}{|c|c|c|}
\hline & Injection & Extraction \\
\hline Energy $T(\mathrm{MeV} / \mathrm{u})$ & 6 & 100 \\
\hline Number of cells $N$ & \multicolumn{2}{|c|}{12} \\
\hline Periodicity & \multicolumn{2}{|c|}{12} \\
\hline Field index $k$ & \multirow{2}{*}{\multicolumn{2}{|c|}{$\begin{array}{c}10.5 \\
(4.81,0.84)\end{array}$}} \\
\hline Tune (horizontal, vertical) & & \\
\hline Ring circumference (m) & 37.964 & 42.995 \\
\hline Frequency (MHz) & 0.894 & 3.002 \\
\hline Acceleration voltage $(\mathrm{kV})$ & \multicolumn{2}{|c|}{18.4} \\
\hline Minimum radius $(\mathrm{m})$ & 5.802 & 6.571 \\
\hline Maximum radius (m) & 6.061 & 6.865 \\
\hline Length of long straight (m) & 0.734 & 0.831 \\
\hline Length of short straight (m) & 0.151 & 0.214 \\
\hline Field strength $(F)(\mathrm{T})$ & 0.392 & 1.450 \\
\hline Field strength $(D)(\mathrm{T})$ & 0.392 & 1.450 \\
\hline Sector angle $\Theta_{F}(F)(\mathrm{deg})$ & \multicolumn{2}{|c|}{15.47} \\
\hline Bending angle $\theta_{F}(F)(\mathrm{deg})$ & \multicolumn{2}{|c|}{48.6} \\
\hline Sector angle $\Theta_{D}(\mathrm{D})(\mathrm{deg})$ & \multicolumn{2}{|c|}{6.13} \\
\hline Bending angle $\theta_{D}$ (D) (deg) & \multicolumn{2}{|c|}{18.6} \\
\hline
\end{tabular}


TABLE VII. Basic lattice parameters of the HE-FFAG ring.

\begin{tabular}{|c|c|c|}
\hline & Injection & Extraction \\
\hline Energy $T(\mathrm{MeV} / \mathrm{u})$ & 100 & 400 \\
\hline Number of cells $N$ & \multicolumn{2}{|c|}{12} \\
\hline Periodicity & \multicolumn{2}{|c|}{12} \\
\hline Field index $k$ & \multicolumn{2}{|c|}{10.5} \\
\hline Tune (horizontal, vertical) & \multicolumn{2}{|c|}{$(4.81,0.83)$} \\
\hline Ring circumference (m) & 64.786 & 69.237 \\
\hline Frequency $(\mathrm{MHz})$ & 1.991 & 3.098 \\
\hline Acceleration voltage $(\mathrm{kV})$ & \multicolumn{2}{|c|}{45.5} \\
\hline Minimum radius $(\mathrm{m})$ & 9.901 & 10.582 \\
\hline Maximum radius $(\mathrm{m})$ & 10.344 & 11.054 \\
\hline Length of long straight (m) & 1.252 & 1.338 \\
\hline Length of short straight (m) & 0.257 & 0.275 \\
\hline Field strength $(F)(\mathrm{T})$ & 0.961 & 1.920 \\
\hline Field strength $(D)(\mathrm{T})$ & 0.962 & 1.932 \\
\hline Sector angle $\Theta_{F}(F)(\operatorname{deg})$ & \multicolumn{2}{|c|}{15.47} \\
\hline Bending angle $\theta_{F}(F)(\mathrm{deg})$ & \multicolumn{2}{|c|}{48.6} \\
\hline Sector angle $\Theta_{D}$ (D) (deg) & \multicolumn{2}{|c|}{6.13} \\
\hline Bending angle $\theta_{D}$ (D) (deg) & \multicolumn{2}{|c|}{18.6} \\
\hline
\end{tabular}

excursion by increasing the field index $k$ as much as possible. For the 12 cell lattice, the maximum possible value of $k$ is about 12 (see Fig. 4). To secure a workable drift space, we have chosen a similar lattice configuration as that of the ME-FFAG (see Table VII). Overall view of the triple-cascade FFAG system is illustrated in Fig. 7 and the $\beta$ functions of each lattice are plotted in Fig. 8. With this design, a ring circumference of $69.2 \mathrm{~m}$ can be realized upon the following conditions and limitations being clarified.

\section{B. Conditions and limitations}

Since there exist several concerns arising from the tight lattice structure, in order to satisfy the required output beam intensity we have further investigated (i) the possibility of achieving a broadband high accelerating gradient rf cavity, (ii) the tune shift due to the fringing field, (iii) an effective fast-extraction system within limited drift space, and (iv) the dynamic aperture of each ring, in order to satisfy the required output beam intensity.

The basic parameters of the rf cavities for each ring are given in Tables V, VI, and VII. As mentioned earlier, we have chosen a soft magnetic alloy such as FINEMET, which has low quality factor and high permeability, to be loaded in an untuned broadband rf cavity. Unlike pulsed synchrotrons, rf cores for FFAG accelerators are required to have a wide physical aperture in the radial direction. In the above triple-cascade scheme, the orbit excursion of each ring is about $80 \mathrm{~cm}$ and we therefore assumed the preliminary core shape of Fig. 9. We have carried out

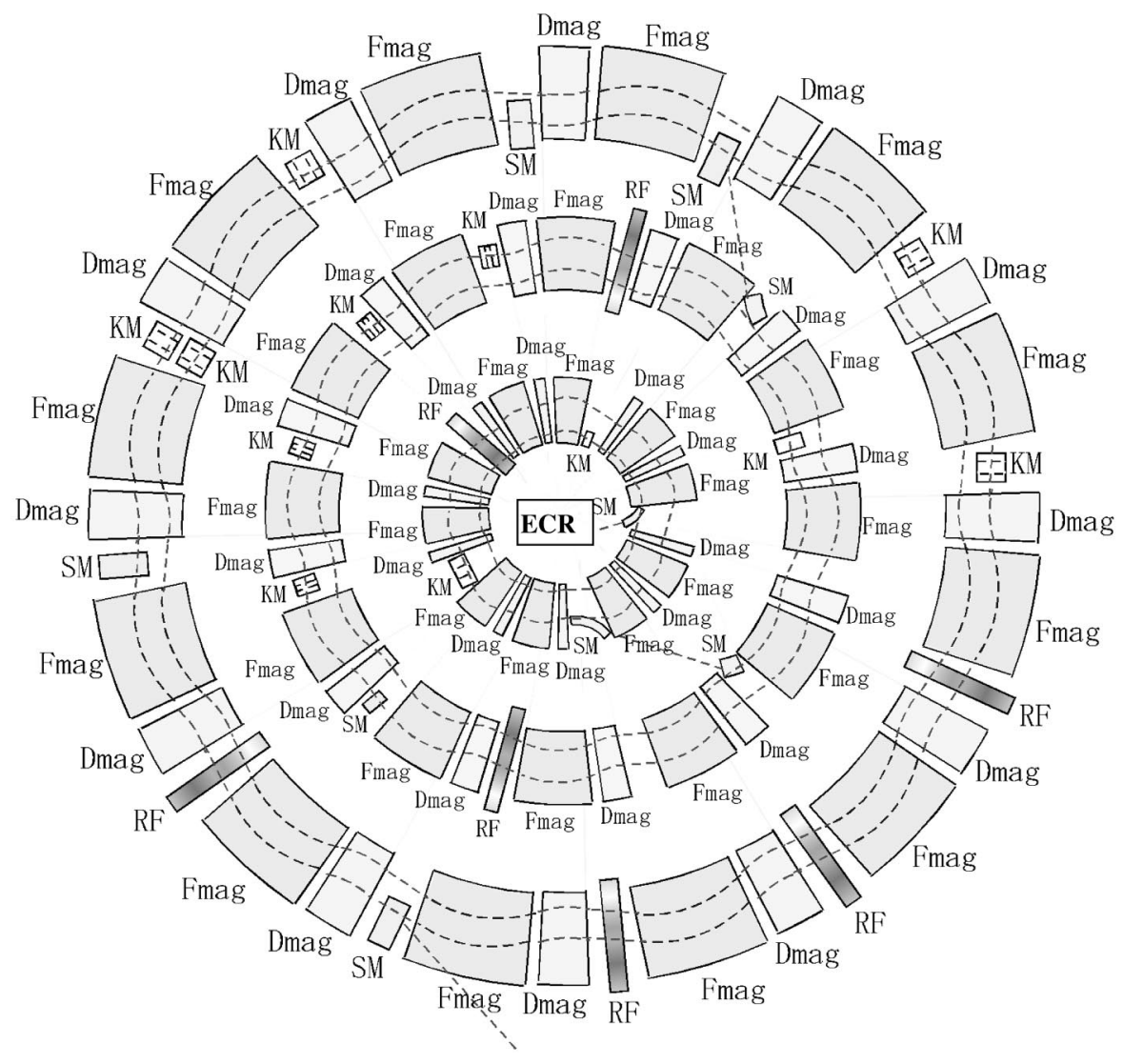

FIG. 7. Overall view of the triple-cascade radial-sector FFAG system. 
(a) LE-FFAG

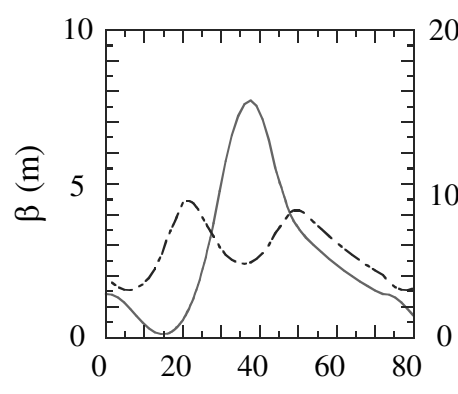

(b) ME-FFAG

(c) HE-FFAG

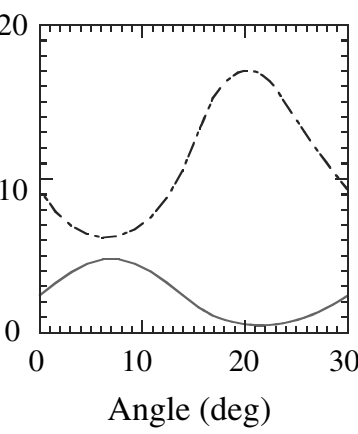

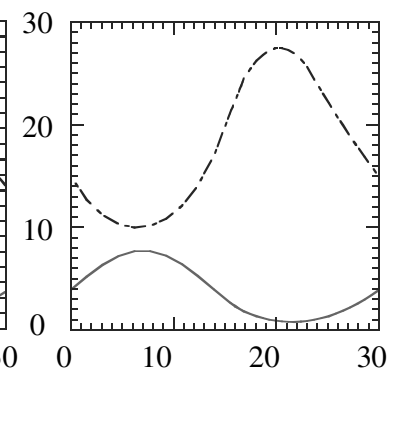

FIG. 8. $\beta$ functions of the (a) LE, (b) ME, and (c) HE rings at extraction orbit.

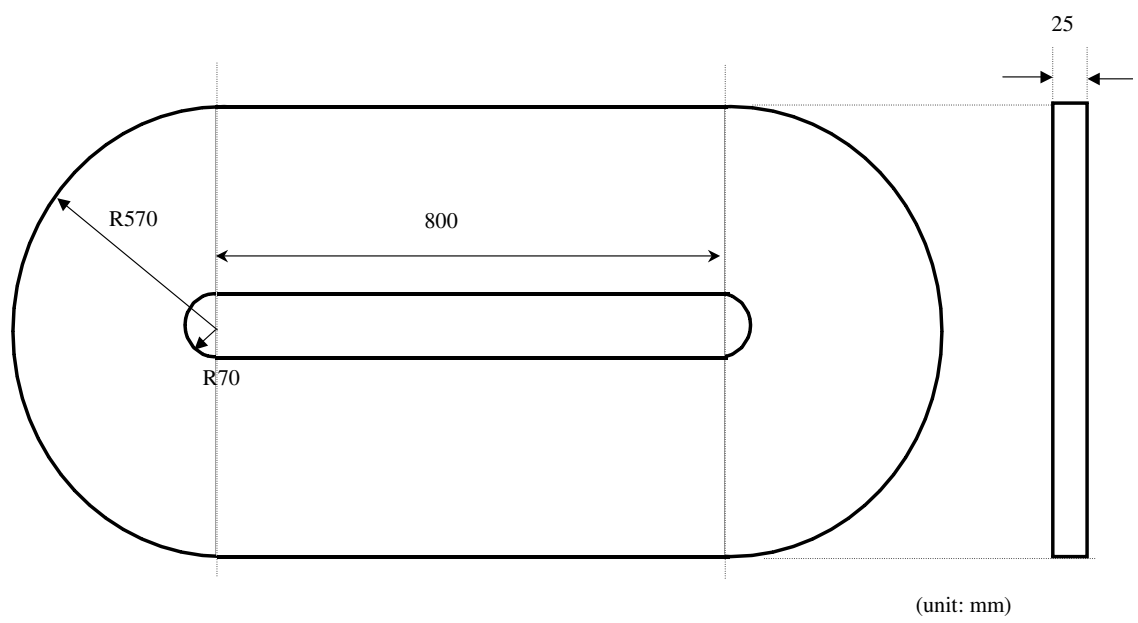

FIG. 9. Racetrack shape of a preliminary MA core.

several measurements to identify the complex permeability of FINEMET and estimated the frequency dependence of the impedance for the racetrack-shaped core, as in Fig. 10. From this estimate, we evaluated a number of cores, total shunt impedance, gap voltage, and required power (see Table VIII). As an example, the frequency dependence of the cavity impedance for the LE ring is also shown in Fig. 11. Although we used a large number of rf cores to reduce the power, due to the high repetition rate of $200 \mathrm{~Hz}$ the total power of rf system is still somewhat large to be considered for a marketable version of a carbon-beam radiotherapy machine

The presence of the fringing field may cause a considerable tune shift. In particular, the effects are expected to be large for the case of the LE-FFAG, since the pole gap varies drastically from injection to extraction orbits due to the large momentum excursion. If we use the linear approximation of the fringing field, the vertical edge matrix $M$ may be written in terms of the gap width $g$, the edge angle $\varepsilon$, and the radius of curvature $\rho$ as

$$
M_{V}^{E}=\left(\begin{array}{ccc}
1 & 0 & 0 \\
-\frac{\tan (\varepsilon-\Psi)}{\rho} & 1 & 0 \\
0 & 0 & 1
\end{array}\right)
$$

with

$$
\Psi=\alpha \frac{g}{\rho} \frac{1+\sin ^{2} \varepsilon}{\cos \varepsilon}
$$

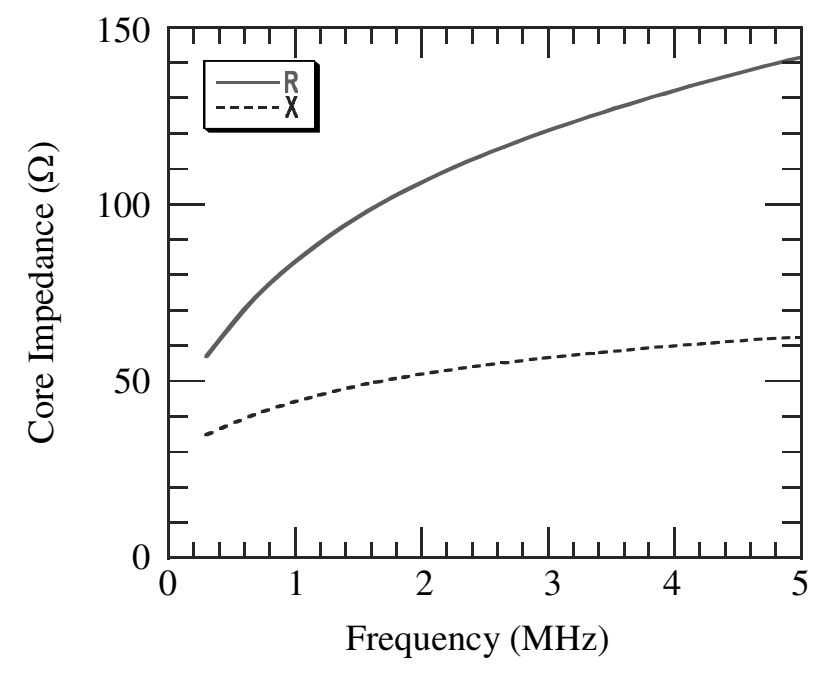

FIG. 10. Frequency dependence of complex impedance $(Z=$ $R+j X)$ of the racetrack-shaped MA core described in Fig. 9. 
TABLE VIII. Basic parameters of rf cavity.

\begin{tabular}{lccc}
\hline \hline \multicolumn{1}{c}{ Parameter } & \multicolumn{2}{c}{ LE-FFAGME-FFAGHE-FFAG } \\
\hline Number of cavities & 1 & 2 & 4 \\
Number of cores per cell & 4 & 10 & 20 \\
Shunt impedance per cell $(k \Omega)$ & 0.34 & 1.22 & 2.86 \\
Total power $(\mathrm{kW})$ & 20 & 90 & 180 \\
\hline \hline
\end{tabular}

Since $g$ and $\rho$ depend on particle momentum, the quantity $g / \rho$ is found to vary by a factor equal to the momentum ratio, implying that the edge angle shift $\Psi$ due to the fringing-field sweeps by factors of 12.27, 4.18, and 2.15 from injection to extraction for the LE, ME, and $\mathrm{HE}$ rings, respectively. Therefore, a severe tune shift is expected for the LE ring. Indeed, the resonance crossing of vertical tunes for the case of the LE ring is predicted by a linear optical calculation with $\alpha=0.48$ (see Fig. 12). Here, a pole gap of $1 \mathrm{~cm}$ is assumed and the vertical tunes at injection are set to the values listed in Table $\mathrm{V}$ by adjusting the bending angles of magnets, for simplicity. Since gradient-field production by pole gap variation is the cause of tune shift, hybrid-type magnets, which produce radial gradient field by combining both coil-current distribution and magnetic pole gap and/or the employment of magnetic clamp at the magnet edge, may be considered to suppress the effect.

Design of the fast-extraction system for the tightly spaced lattice configuration is also a problem, especially for the HE-FFAG ring. We have estimated that eight kickers of Table IX and two septum magnets of Table X are needed for the HE ring to achieve a beam separation of $420 \mathrm{~mm}$, as shown in Fig. 13. In this case, the extracted beam must go through the return yoke of a main magnet. If we attempted to ease the extraction system, then extending the drift length by increasing the ring circumference is inevitable.

Finally, we evaluated the dynamic apertures for the three rings by performing 1000 turns of particle tracking

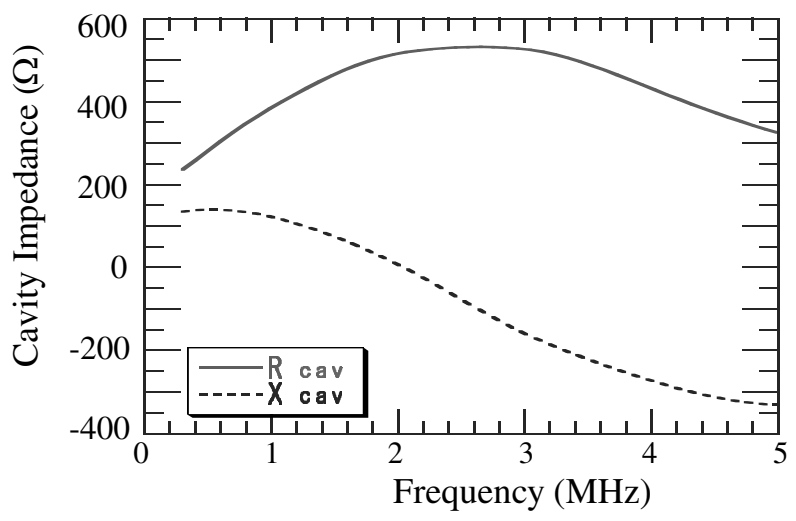

FIG. 11. Frequency dependence of the cavity impedance $\left(Z_{\text {cav }}=R_{\text {cav }}+j X_{\text {cav }}\right)$ for the LE-FFAG ring. Here, four cores per cell and a gap capacitance of $70 \mathrm{pF}$ are assumed.

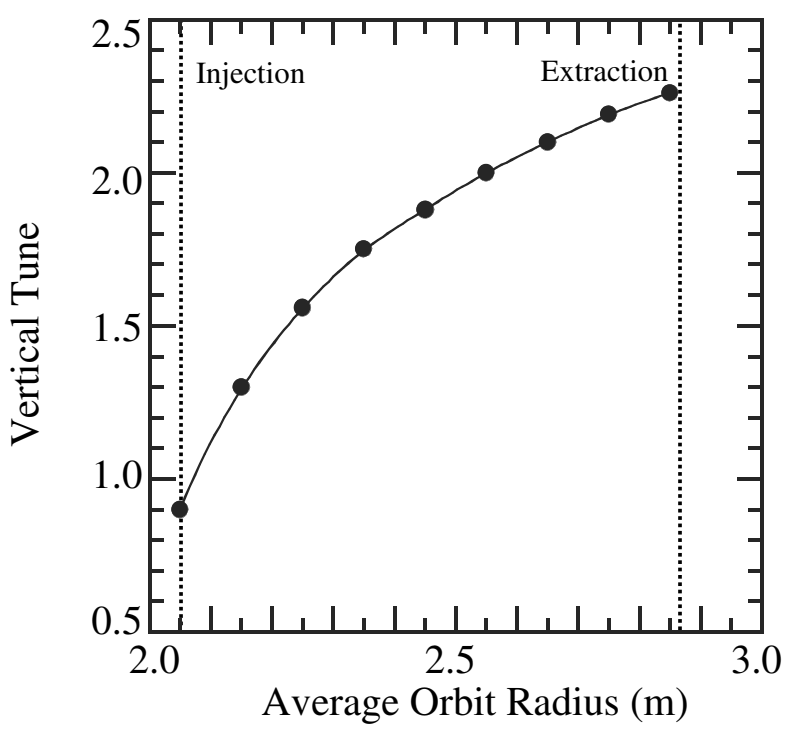

FIG. 12. Vertical tune shifts for the case of the LE ring. A pole gap of $1 \mathrm{~cm}$ is assumed and the vertical tune at injection is adjusted to the value listed in Table V, using the linear optical model described in the text.

under square edge field assumption; the simulation results are shown in Fig. 14. According to Table III, the beam emittances for the LE, ME, and HE rings are 108, 8.8, and $2.1 \pi \mathrm{mm}$ mrad, respectively. The dynamic apertures for both $\mathrm{ME}$ and $\mathrm{HE}$ rings are large enough to accept the

TABLE IX. Parameters of kicker magnets used to estimate the fast-extraction system of the HE-FFAG.

\begin{tabular}{lc}
\hline \hline & KCR3-E1-E8 \\
\hline Number of kicker magnets & 8 \\
Bending angle (mrad) & 7.5 \\
Pole length (mm) & 450 \\
Pole width (mm) & 60 \\
Gap width (mm) & 30 \\
Magnetic field (T) & 0.1 \\
Raising time (ns) & 454 \\
Flattop (ns) & 200 \\
\hline \hline
\end{tabular}

TABLE X. Parameters of septum magnets used to estimate the fast-extraction system of the HE-FFAG.

\begin{tabular}{lcc}
\hline \hline & SM3-E1 & SM3-E2 \\
\hline Number of septum magnets & 1 & 1 \\
Bending angle (mrad) & 3.071 & 12.536 \\
Pole length (mm) & 570 & 1160 \\
Pole width (mm) & 50 & 50 \\
Gap width (mm) & 30 & 30 \\
Magnetic field (T) & 0.8 & 1.2 \\
Septum width (mm) & 48 & 60 \\
\hline \hline
\end{tabular}




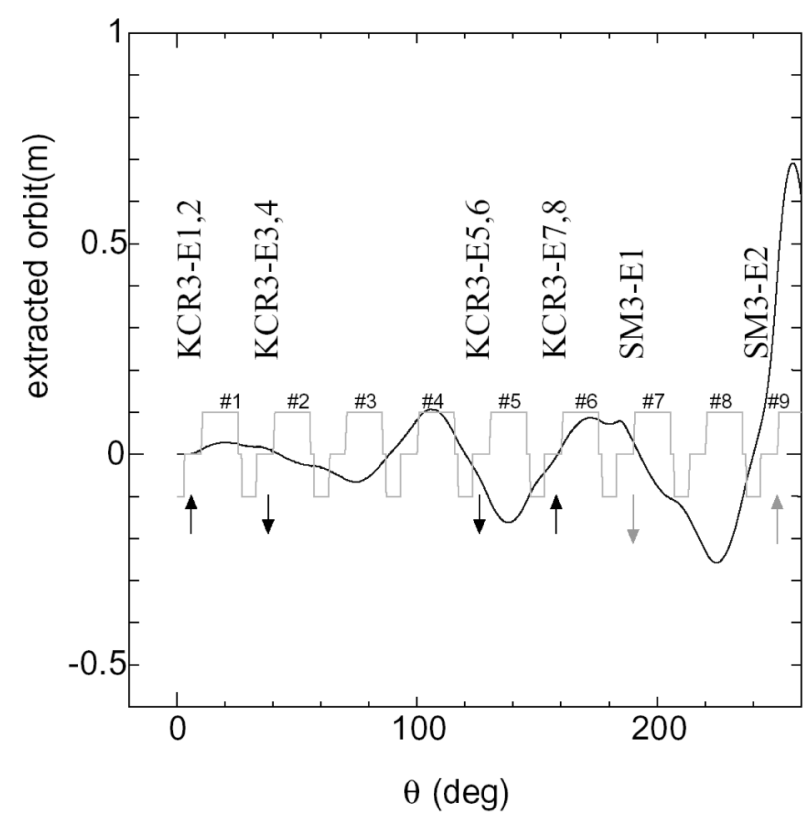

FIG. 13. Fast-extraction system estimated for tightly spaced lattice configuration of the HE-FFAG ring. Assuming bruteforce extraction, at least eight kickers and two septum magnets are needed to obtain a beam separation of $420 \mathrm{~mm}$. required emittance, whereas the dynamic aperture for the LE ring is too small and leads to beam loss. For this reason, we have estimated the transmission rate during the acceleration process in the LE ring as $20 \%$, as shown in Table IV, but the required beam intensity can still be guaranteed by increasing the injection beam pulse width for the LE-FFAG to $1.4 \mu \mathrm{s}$.

\section{SUMMARY}

We have studied various lattice structures in order to design a compact, cost-effective medical FFAG accelerator for carbon-beam cancer therapy.

For the radial-sector configuration, minimizing $C$ and maximizing $k$ are essential to design a compact machine but cannot be achieved at the same time. In this study, some of the stability regions were surveyed to search for the appropriate cell number $N$ that gives the maximum value of $k$, while keeping the ring circumference less than $70 \mathrm{~m}$. An important general result is that viable radialsector designs are possible with circumference factors $C$ significantly lower than the value 4.45 previously quoted. Our preliminary design shows a radial-sector FFAG consisting of triple-cascade rings, in which both focusing and defocusing magnets are combined in one to compose a

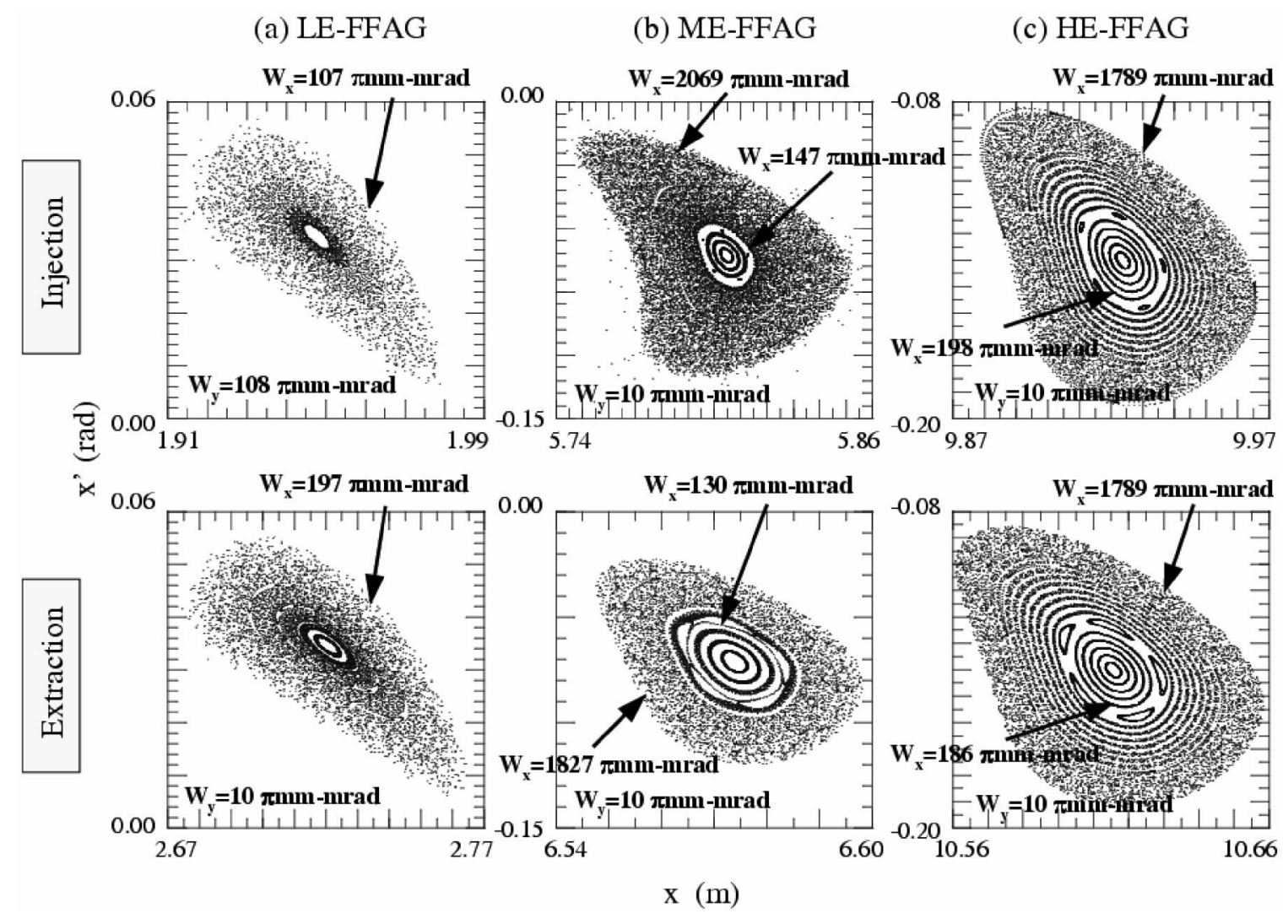

FIG. 14. Dynamic apertures for the (a) LE, (b) ME, and (c) HE rings. Top and bottom figures correspond to injection and extraction orbits, respectively. 1000 turns of tracking are performed. 
main sector magnet and the orbit excursion is kept below $80 \mathrm{~cm}$. A ring circumference of $69.2 \mathrm{~m}$ (corresponding to $C=3.3$ ), which we believe is nearly the optimum size for the radial-sector type, is realized with normal conducting magnets of $1.93 \mathrm{~T}$.

On the other hand, minimizing $C$ is no longer a major task for the spiral-sector type. Instead, maximizing $k$ and minimizing $\xi$ become a primary consideration. In order to design a double-cascade accelerating scheme with spiral sectors, the spiral angle $\xi$ needs to be at least $65^{\circ}$. Considering the shallow orbital stability at higher values of $\xi$, the complexity of producing radial gradient-field distributions with sharp spiral edge, field imperfections, and the clearance of rf cavity setup, etc., highly spiraled configurations were not considered as a choice in our present study.

According to the triple-cascade radial-sector design, we also investigated (i) the possibility of achieving a broadband high accelerating gradient rf cavity, (ii) the tune shift due to the presence of the fringing field, (iii) a fast-extraction system within limited drift space, and (iv) the dynamic apertures of each ring. Design of a broadband rf cavity is regarded as possible using low- $Q$ high-permeability MA cores, although a large number of cores is necessary to achieve the high repetition rate of $200 \mathrm{~Hz}$. For the LE ring, the radial field gradient may not be obtained with pole gaps because of the severe tune shift due to the presence of the fringing field. To avoid the fringing effect, design of a hybrid-type magnet, in which radial gradient field is produced by a coil-current distribution near the injection orbit and by a magnetic pole near the extraction orbit, could be considered. The fastextraction system was investigated and a beam separation of $420 \mathrm{~mm}$ can be achieved with a large number of extraction components. Considerable beam loss is expected for the LE ring due to its small dynamic aperture, and its beam transmission rate during acceleration was estimated as low as $20 \%$. In this sense, the lattice design shown in Fig. 8 is not yet fully optimized, and further design study may lead to larger machines. We conclude that the ring circumference of about $70 \mathrm{~m}$ is nearly as compact as we can expect for a normal-conducting radial-sector FFAG.

\section{ACKNOWLEDGMENTS}

The authors express their cordial thanks to the members of the Accelerator Physics and Engineering Division at NIRS for helping their research advancement. Support from Hitachi Metal Co., Mitsubishi Electronics Co., and Toshiba Co., as well as fruitful discussions with the KEK group, is also greatly appreciated. This work has been supported by the Ministry of Education, Culture, Sports, Science and Technology.

[1] S. Yamada, in Proceedings of the 2nd Asian Particle Accelerator Conference, Beijing, China, 2001, edited by W. B. Liu et al., (Institute of High Energy Physics Chinese Academy of Sciences, Beijing, 2001), p. 829.

[2] T. Misu et al., in Proceedings of the 8th European Particle Accelerator Conference, Paris, 2002, edited by T. Garvey et al., (EPS-IGA and CERN, Geneva, 2002), p. 599.

[3] T. Kanai, K. Kawachi, Y. Kumamoto, H. Ogawa, T. Yamada, H. Matsuzawa, and T. Inada, Med. Phys. 7, 365 (1980).

[4] F.T. Cole, R. O. Haxby, L.W. Jones, C. H. Pruett, and K. M. Terwilliger, Rev. Sci. Instrum. 28, 403 (1957).

[5] M. Aiba et al., in Proceedings of the 7th European Particle Accelerator Conference, Vienna, 2000 (European Physical Society, Geneva, 2000), p. 581; Y. Mori et al., in Proceedings of the 12th Symposium on Accelerator Science and Technology, Wako, Japan, 1999 (RIKEN, Wako, 1999), p. 81.

[6] M. Aiba et al., in Proceedings of the 8th European Particle Accelerator Conference, Paris, 2002 (Ref. [2]), p. 1028.

[7] D. Normile, Science 302, 379 (2003), http://www.rri.kyoto-u.ac.jp/KART/index/img/Science_Magazine.pdf.

[8] Y. Mori, in Proceedings of the 7th European Particle Accelerator Conference, Vienna, 2000 (Ref. [5]), p. 289.

[9] C. Johnstone and S. Koscielniak, Nucl. Instrum. Methods Phys. Res., Sect. A 503, 445 (2003).

[10] K. R. Symon, D.W. Kerst, L.W. Jones, L. J. Laslett, and K. M. Terwilliger, Phys. Rev. 103, 1837 (1956).

[11] J. J. Livingood, Principles of Cyclic Particle Accelerators (Van Nostrand, Princeton, 1961). 